\title{
The Use of Aspiration Catheter Systems for Embolic Protection during Intracranial Vertebral Artery Angioplasty and Stenting
}

\author{
Silvia I. Gesheva ${ }^{a} \quad$ Laurel H. Hastings $^{b}$ Jason D. Wilson ${ }^{a}$ \\ Departments of a Neurosurgery and b Surgery, Louisiana State University Health Sciences Center \\ New Orleans, New Orleans, La., USA
}

\section{Key Words}

Atherosclerosis · Embolic protection device $\cdot$ Stroke $\cdot$ Vertebrobasilar insufficiency

\begin{abstract}
Background: Posterior circulation strokes comprise approximately $20-25 \%$ of all strokes of ischemic origin. Strokes affecting this area carry a significantly higher risk for subsequent stroke or death as compared to anterior circulation strokes. Embolic protection device (EPD) use for carotid artery stenosis has translated into percutaneous interventions of proximal vertebral artery (VA) stenosis. However, the use of EPDs when treating intracranial lesions has yet to be studied and may not be feasible as the vessel caliber is frequently smaller than in existing devices. Objective: The aim of this study is to describe a proximal aspiration technique used during the treatment of intracranial VA and basilar artery (BA) atherosclerotic disease. Methods: Proximal embolic protection was utilized during the treatment of intracranial VA/ BA stenosis with angioplasty and stenting in patients with medically refractory disease. Results: Three patients with severe symptomatic posterior circulation stenosis refractory to medical management were treated with angioplasty and stenting utilizing proximal aspiration. Pre- and post-treatment angiograms and MRIs were compared. Treated vascular stenoses were significantly improved, without new neurological deficits or ischemic injury identified on imaging. Conclusions: The proposed technique of proximal embolic protection may help overcome the challenge of embolus propagation inherent to the treatment modality that was encountered during the treatment of intracranial VA/BA stenosis.

(C) 2016 S. Karger AG, Basel
\end{abstract}




\section{Introduction}

Atherosclerotic disease is a major cause of ischemic events worldwide [1-3]. Posterior circulation strokes comprise approximately $20-25 \%$ of all strokes of ischemic origin. Strokes affecting this area carry a significantly higher risk for subsequent stroke or death as compared to anterior circulation strokes in the acute phase [7 days following initial stroke or transient ischemic attack (TIA)] [4, 5].

Carotid artery stenosis has been treated with angioplasty and stenting for several decades [6-8]. One of the major concerns with these procedures is the risk of embolic sequelae following percutaneous angioplasty and stenting. The distal embolic protection device (EPD) and the proximal cerebral protection Mo.Ma Ultra device (Medtronic, Memphis, Tenn., USA) were both developed and utilized to mitigate this risk $[7,9,10]$. Additionally, the Centers for Medicaid \& Medicare Services (CMS) require the use of EPDs for procedure reimbursement [11].

Due to the evidentiary support for protection devices in the treatment of carotid artery lesions, EPD use has translated into percutaneous interventions of proximal vertebral artery (VA) stenosis $[9,12]$. Lesions of a VA origin and at the V1 segment lend themselves well for distal embolic protection as vessel size approximates the coronaries; there are several devices available for use. However, the use of EPDs when treating intracranial lesions has yet to be studied, and may not be feasible as the vessel caliber is frequently smaller than in existing devices.

The use of EPDs has been described in several studies involving angioplasty and stenting of lesions of a VA origin $[1,5,7,9,10,12-14]$. To date, newer or alternative methods for cerebral protection for the treatment of vertebrobasilar (VB) stenosis have not yet been elucidated. Aspiration systems are currently in use for recanalization of large vessel occlusions in ischemic stroke and may represent an additional method for reducing posterior circulation emboli from stenting and angioplasty.

\section{Methods}

Three patients with medically refractory VB stenosis were treated preemptively with the Penumbra aspiration system during VB angioplasty and stenting. Pre- and post-treatment angiograms and MRIs were analyzed for vessel occlusion and new ischemic injury. Follow-up modified Rankin Scale (mRS) scores were also recorded at discharge and the 3- and 6-month clinic visits.

Procedure in Detail

All patients were placed on aspirin $325 \mathrm{mg}$ and clopidogrel $75 \mathrm{mg}$ daily prior to the procedure and were continued on this regimen postoperatively. Using the Seldinger technique, an 11-cm 6-Fr Cordis sheath was placed in the femoral artery. Using a 5-Fr DAV catheter (Cook Medical Inc., Bloomington, Ind., USA), a diagnostic angiogram was performed. Heparin boluses $(70 \mathrm{U} / \mathrm{kg})$ were given following the diagnostic portion of the angiogram. Activated clotting time was maintained between 250 and $300 \mathrm{~s}$ throughout the procedure.

Following acquisition of imaging, the DAV catheter was then exchanged for a 0.070 6-Fr Neuron catheter (Penumbra Inc., Alameda, Calif., USA) using a Rosen Exchange $0.035 \times 260$ wire (Boston Scientific Corporation, Quincy, Mass., USA) in the subclavian artery. The Neuron catheter was then navigated as distally as possible in the target VA (V3 segment) proximal to the area of stenosis. Distal intracranial access was achieved with a Transcend Floppy 0.014 300-cm exchange-length wire (Boston Scientific Corporation). Proximal aspiration through the 6-Fr Neuron was achieved using the Penumbra aspiration pump. In one patient (case 1), aspiration was performed continuously during the entire procedure, resulting in a blood loss of $700 \mathrm{ml}$. Our technique was subsequently modified; in the rest of the cohort, Penumbra aspiration was only utilized while traversing the stenosis and during angioplasty and stenting, thus minimizing the blood loss to $<150 \mathrm{ml}$. Following the intervention, pre- and post-treatment angiographic runs were compared. Post-treatment MRIs were acquired within $24 \mathrm{~h}$. 

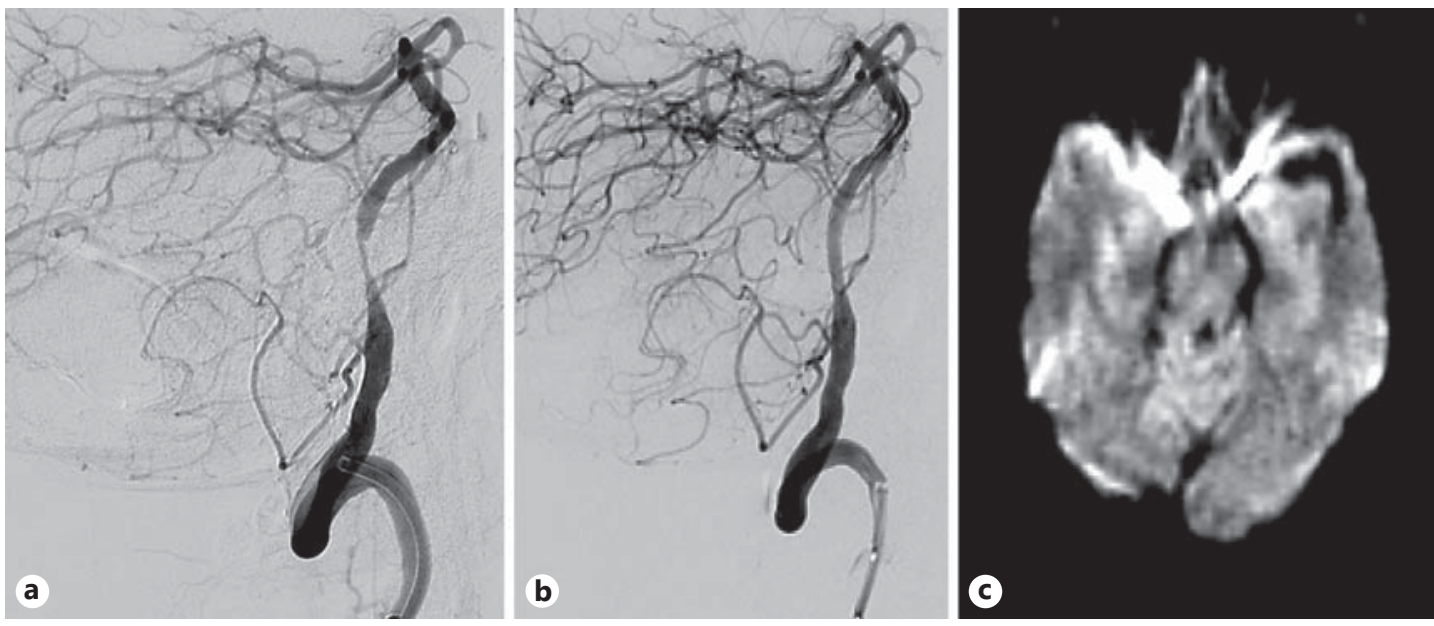

Fig. 1. a Lateral pretreatment cerebral angiogram. b Lateral post-treatment cerebral angiogram. c Post-treatment diffusion-weighted MRI.

\section{Results}

\section{Case 1}

The patient was a 68-year-old female with a history of hypertension and a prior cerebrovascular accident with residual left-sided hemiparesis who presented with worsening dysarthria, dizziness and difficulty in balance refractory to medical management. Magnetic resonance angiography performed during evaluation confirmed severe stenosis of the right VB junction. On angiography, the left VA was hypoplastic with 80\% stenosis of the V3 segment and termination in the left posterior inferior cerebellar artery. We were unsuccessful at accessing the right VA through a femoral approach; therefore, a 4-cm 6-Fr Cordis sheath was placed in the right brachial artery. The VA was selected with a 6-F Neuron catheter. Imaging revealed severe stenosis, $>90 \%$, of the V4 segment at the VB junction (fig. 1b). Our operative steps and supplies used were identical to the first patient's procedure; however, the Penumbra aspiration system was used continuously throughout the procedure, resulting in a 700-ml estimated blood loss. Post-treatment angiography demonstrated no residual stenosis (fig. 1c). Pre- and post-treatment MRI demonstrated stable areas of diffusion restriction in the anterior pons and bilateral cerebellar hemispheres (fig. 1a, c).

\section{Case 2}

The second patient was a 57-year-old male with a history of VB stenosis and posterior circulation stroke initially conservatively managed (aspirin/Plavix). He was readmitted after several recurrent episodes of dizziness and unsteady gait refractory to medical management. A cerebral angiogram demonstrated a dominant left VA with $60 \%$ stenosis of the V1 segment and $80-90 \%$ stenosis of the basilar artery (BA; fig. 2a). The 6-F Neuron catheter (Penumbra Inc.) was navigated to the distal V3 segment of the VA. Distal intracranial access was achieved with a Transcend Floppy $0.014300-\mathrm{cm}$ exchange-length wire (Boston Scientific), which was maintained in the distal posterior cerebellar artery. Pre-stent dilatation was performed with a $2.0 \times 20-\mathrm{mm}$ Gateway PTA balloon (Stryker Corporation, Kalamazoo, Mich., USA). Proximal aspiration utilizing the Penumbra aspiration system (Penumbra Inc.) attached to the base catheter was performed during manipulation of the stenosis (angioplasty and stenting). Following angioplasty, a Wingspan Stent $4.5 \times 20 \mathrm{~mm}$ (Boston Scientific) was deployed across the area of stenosis under continuous proximal aspiration. Post-stent angioplasty was 
Gesheva et al.: The Use of Aspiration Catheter Systems for Embolic Protection during Intracranial Vertebral Artery Angioplasty and Stenting
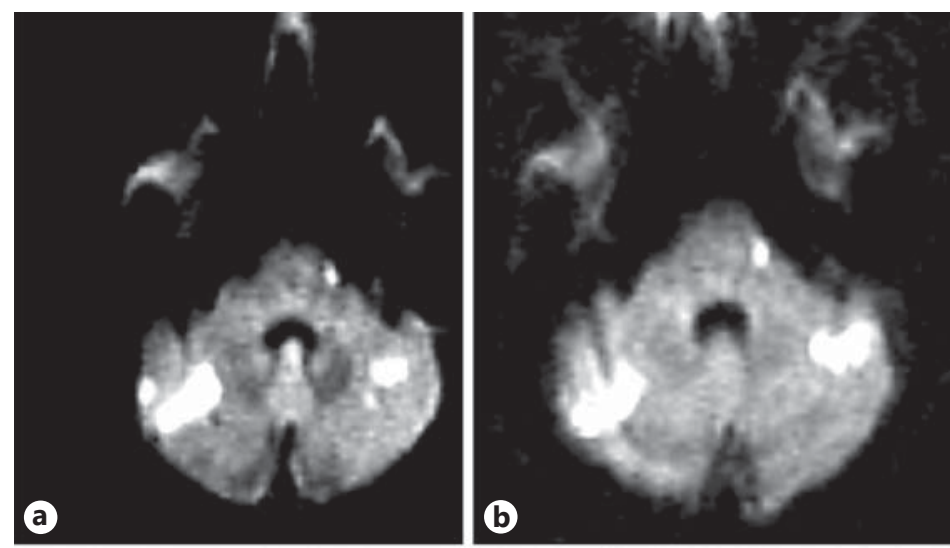

Fig. 2. a Pretreatment diffusionweighted MRI. b Post-treatment diffusion-weighted MRI. c Pretreatment anteroposterior (AP) cerebral angiogram. d Post-treatment AP cerebral angiogram.

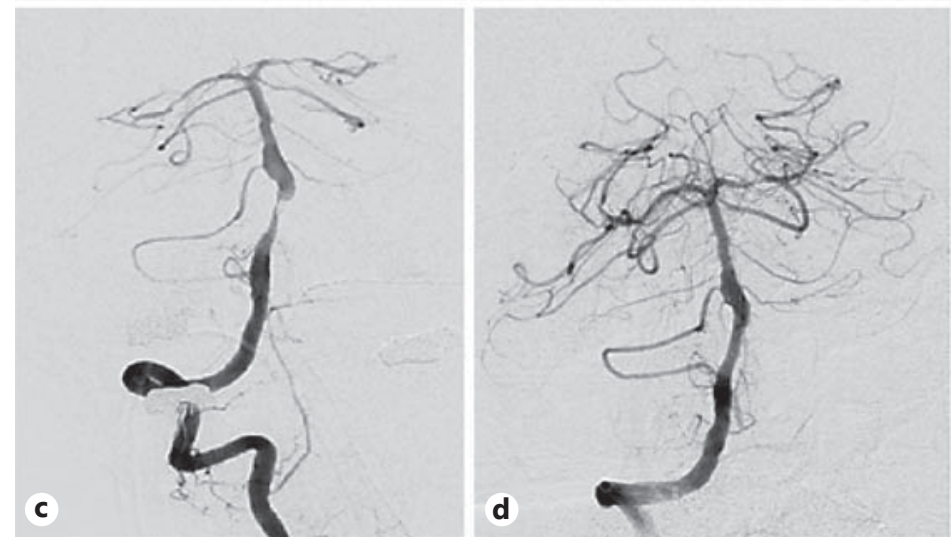

performed with minimal residual stenosis (fig. 2b). Delayed imaging confirmed no platelet aggregation. Following the procedure, a MynxGrip closure device (Cardinal Health, Dublin, Ohio, USA) was placed. Post-treatment MRI demonstrated no new area of diffusion restriction (fig. 2c). At discharge and the 3- and 6-month follow-ups, the patient's mRS score was 0. Follow-up angiography was performed at 6 months, showing $>70 \%$ in-stent stenosis; however, at the scheduled intervention date, repeat imaging showed improvement to $<50 \%$ stenosis. This was attributed to the fact that the patient was an intermediate metabolizer of clopidogrel, which required raising the oral dose in order to increase the plasma levels of active metabolites [15].

\section{Case 3}

The last patient in our case series was a 75-year-old, right-handed male with a history of stroke (right face, arm and leg weakness). Magnetic resonance angiography images revealed severe basilar stenosis. The patient was managed conservatively with aspirin, Plavix and a statin. He was readmitted 2 months later with recurrent symptoms. Pretreatment MRI demonstrated a new area of diffusion restriction in the pons (fig. 3d). A cerebral angiogram revealed an isolated posterior circulation and $>90 \%$ stenosis of the distal basilar artery with minimal anterograde contrast penetration (fig. 3a-c). Following placement of the Neuron catheter in the V4 segment of the right VA, the procedure was performed under proximal aspiration. Pre-stent dilatation was performed with a $1.5 \times 9-\mathrm{mm}$ Gateway balloon (Stryker Corporation). This was followed by a $2.5 \times 20$-mm Wingspan stent (Stryker Corporation). Final angiographic imaging revealed a significant improvement in vessel filling (fig. $3 c, d$ ). This patient was followed as an outpatient at 3 and 6 months, with a mRS score of 0 at both 

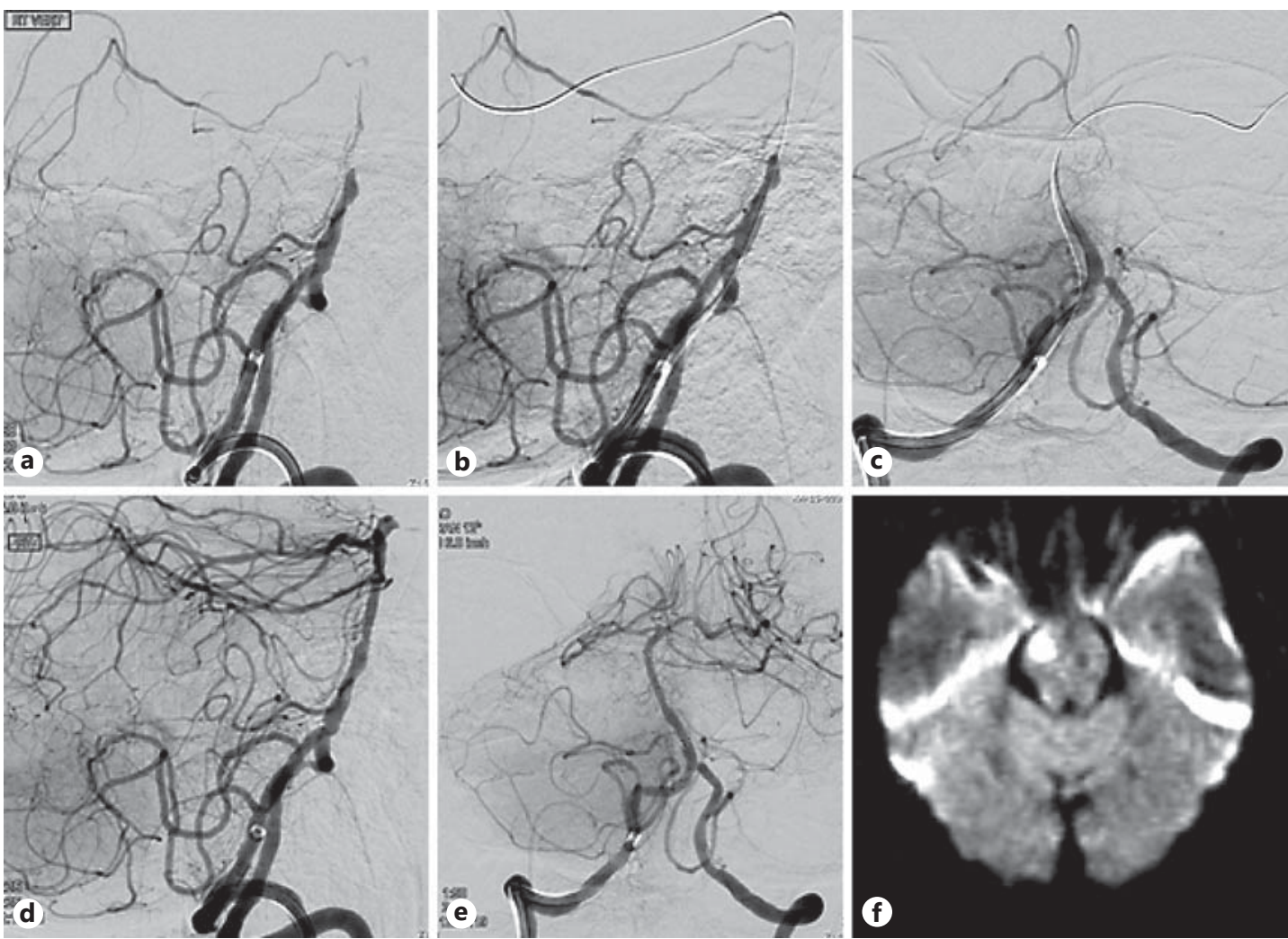

Fig. 3. a, b Lateral pretreatment cerebral angiograms. c Anteroposterior (AP) pretreatment cerebral angiogram. d, e AP and lateral post-treatment cerebral angiograms. f Post-treatment diffusion-weighted MRI.

visits and only complaining of residual hand paresthesia. No additional angiography has been performed to date.

All patients were transferred to the intensive care unit postoperatively for monitoring and received a brain MRI on post-procedural day 1 to detect the presence of new areas of diffusion restriction. Follow-up mRS scores in all patients were $0-1$. Repeat angiography was performed at 6 months on all patients in the study to evaluate for restenosis or disease progression.

\section{Discussion}

Various studies have examined intracranial stenosis and the endovascular treatment of VB disease. They have demonstrated that in the acute phase, up to 7 days following a posterior circulation TIA or stroke, the risk of subsequent stroke or death is significantly increased as compared to anterior circulation strokes. Eberhardt et al. [16] reported that after VB TIA or stroke, the risk for subsequent stroke over the following 5 years is 22-35\% [17]. The WASID trial demonstrated annual stroke rates for patients with symptomatic intracranial vertebral or BA stenosis at 7.8 and $10.7 \%$, respectively [3].

Endovascular interventions are currently being undertaken in patients with symptomatic VB intracranial stenosis refractory to medical treatment. Proximal vertebral lesions lend themselves well to distal EPDs. Conversely, due to vessel size and lesional configuration, EPDs are not typically utilized in distal vertebral or BA disease, and endovascular inter- 
vention may lead to subsequent cerebrovascular events. Canyigit et al. [6] reported that 29\% of the study cases had diffusion-weighted imaging abnormalities after the procedure. Neurologic complication rates vary widely based on the current literature (from 0 to $23 \%$ ), likely secondary to the limited number of studies that have been conducted on intracranial stenting of VB lesions.

The use of the Penumbra aspiration system during vertebral stenting has not been described to date. As a result of our series, we now use proximal aspiration only with manipulation of the stenotic lesion to reduce total blood loss, as encountered in our second patient. Finally, proximal aspiration can be applied to any base/therapeutic catheter [e.g., 90-cm 6-F Envoy (Codman Neuro, Raynham, Mass., USA)]. We have applied this technique during the treatment of proximal VA stenosis in an additional patient, who required catheter placement in the VA ostia.

Although our limited retrospective series requires further prospective validation, we claim that the risk profile for the utilization of this proximal aspiration technique is extremely low. As flexible catheters become more navigable, we are able to achieve distal intracranial access with larger-diameter catheters, which lend themselves to proximal aspiration. Future catheter generations may also include balloon options that could theoretically improve flow reversal and embolic protection.

\section{Conclusions}

Due to recent advancements in endovascular therapy, intracranial VB stenoses have become treatable with endovascular angioplasty and stenting. Current recommendations advise angioplasty and stenting in symptomatic high-grade stenosis of $>70 \%$ in patients whose symptoms are refractory to medical management [18]. The use of an aspiration system selectively during angioplasty and stent deployment may reduce clinically relevant periprocedural emboli and stroke risk. We realize that proximal aspiration cannot eliminate the documented stroke risk of $8.2 \%$ that is associated with perforator occlusion [19]. However, a decrease in embolic phenomena could have profound effects on both patients' quality of life and health care utilization resulting from the morbidity and mortality associated with VB stenosis and its management.

\section{Acknowledgement}

The authors would like to thank Erin Fannin for her editorial support during manuscript preparation.

\section{Disclosure Statement}

The authors have no personal or institutional financial interest in the materials described in this paper.

\section{References}

1 Mohammadian R, Sharifipour E, Mansourizadeh R, et al: Angioplasty and stenting of symptomatic vertebral artery stenosis. Neuroradiol J 2013;26:454-463.

2 SSYLVIA Study Investigators: Stenting of Symptomatic Atherosclerotic Lesions in the Vertebral or Intracranial Arteries (SSYLVIA): study results. Stroke 2004;35:1388-1392.

3 Warfarin-Aspirin Symptomatic Intracranial Disease (WASID) Study Group: Prognosis of patients with symptomatic vertebral or basilar artery stenosis. Stroke 1998;29:1389-1392. 
Gesheva et al:: The Use of Aspiration Catheter Systems for Embolic Protection during

4 Abuzinadah AR, Akabazt MH, Almekhlafi MA, et al: Stroke recurrence rates among patients with symptomatic intracranial vertebrobasilar stenoses: systematic review and meta-analysis. J Neurointerv Surg 2014, Epub ahead of print.

5 Coward LJ, Featherstone RL, Brown MM: Percutaneous transluminal angioplasty and stenting for vertebral artery Stenosis. Cochrane Database Syst Rev 2005;CD000516.

6 Canyigit M, Arat A, Cil BE, et al: Distal embolization after stenting of the vertebral artery: diffusion-weighted magnetic resonance imaging findings. Cardiovasc Intervent Radiol 2007;30:189-195.

7 Coward LJ, McCabe DJ, Ederle J, et al: Long-term outcome after angioplasty and stenting for symptomatic vertebral artery stenosis compared with medical treatment in the Carotid and Vertebral Artery Transluminal Angioplasty Study (CAVATAS). Stroke 2007;38:1526-1530.

8 Chimowitz MI, Lynn MJ, Derdeyn CP, et al: Stenting versus aggressive medical therapy for intracranial arterial stenosis. N Engl J Med 2011;365:993-1003.

9 Qureshi A, Kirmani J, Harris-Lane P, et al: Vertebral artery origin stent placement with distal protection: technical and clinical results. AJNR Am J Neuroradiol 2006;27:1140-1145.

10 Sharma S, Bhambi B: Vertebral artery stenoting utilizing distal embolic protection with Filter wire and a drugeluting Taxus Stent. Acute Card Care 2006;8:235-237.

11 Centers for Medicare \& Medicaid Services: National coverage determination (NCD) for percutaneous transluminal angioplasty (PTA) (20.7). https://www.cms.gov/medicare-coverage-database/details (accessed May 10, 2014).

12 Mintz EP, Gruberg L, Kouperberg E, et al: Vertebral artery stenting using distal emboli protection and transcranial Doppler. Catheter Cardiovasc Interv 2004;61:12-15.

13 Flossman E, Rothwell PM: Prognosis of vertebrobasilar transient ischaemic attack and minor stroke. Brain 2003;126:1940-1954.

14 Sawada M, Hashimoto N, Nishi S, et al: Detection of embolic signals during and after percutaneous transluminal angioplasty of subclavian and vertebral arteries using transcranial Doppler ultrasonography. Neurosurgery 1997;41:535-541.

15 LabCorp, Laboratory Corporation of America Holdings 2014: A technical review: clopidogrel CYP2C19 genotyping. https://www.labcorp.com/.EdosPortlet/TestMenuLibrary (accessed November 15, 2015)

16 Eberhardt O, Naegele T, Raygrotzki S, Weller M, Ernemann U: Stenting of vertebrobasilar arteries in symptomatic atherosclerotic disease and acute occlusion: case series and review of literature. J Vasc Surg 2006;43: 1145-1154.

17 Heyman A, Wilkinson WE, Hurwitz BJ, et al: Clinical and epidemiologic aspects of vertebrobasilar and nonfocal cerebral ischemia; in Berguer R, Bauer RB (eds): Vertebrobasilar Arterial Occlusive Disease. Medical and Surgical Management. New York, Raven Press, 1984, pp 27-36.

18 US Food and Drug Administration: Narrowed indications for use for the Stryker Wingspan Stent System: FDA safety communication. http://www.fda.gov/MedicalDevices/Safety/AlertsandNotices/ucm314600.htm (accessed September 10, 2013).

19 Jiang WJ, Srivastava T, Gao F, et al: Perforator stroke after elective stenting of symptomatic intracranial stenosis. Neurology 2006;66:1868-1872. 